\title{
EDIFICIO "IBM AVENIDA DE AMÉRICA" MADRID-ESPAÑA
}

\author{
(IBM BUILDING AT AVENIDA OF AMERICA. MADRID/SPAIN)
}

Salvador Gayarre, Tomás Domínguez y Juan Martín Baranda, Arquitectos

Fecha de recepción: 23-IV-91

\section{RESUMEN}

El tema muy actual de los "edificios inteligentes", es decir, los que incorporan elementos de las Tecnologías de la Información que los capaciten para la prestación de servicios avanzados, es tratado en el presente artículo referido a un magnifico edificio madrileño, concebido para ayudar al individuo, tanto a las personas que trabajan en él como a aquellas que mantienen relación con IBM España.

\section{Se sintetizan en el artículo las características} arquitectónicas del edificio y se describen los sistemas y servicios más importantes, incluyéndose las plantas y perspectivas que permiten al lector tener una idea del conjunto.

\section{SUMMARY}

The very actual subject of the "smart builgings", as those that incorporate information technologie elements to be able for the avanced services performances, is the theme of this work about the IBM building in Madrid, that has been thought to help the persons that work behind it or that have any relations hip with the enterprise.

The main architectural characteristics systems and services are described, including perspectives and drawings to make easier the comprehension of the proyect.

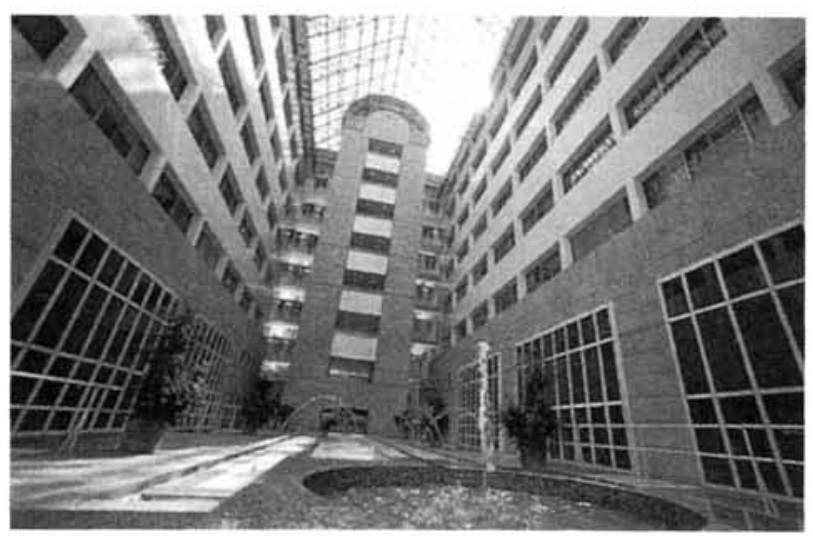

(C) Consejo Superior de Investigaciones Científicas Licencia Creative Commons 3.0 España (by-nc)

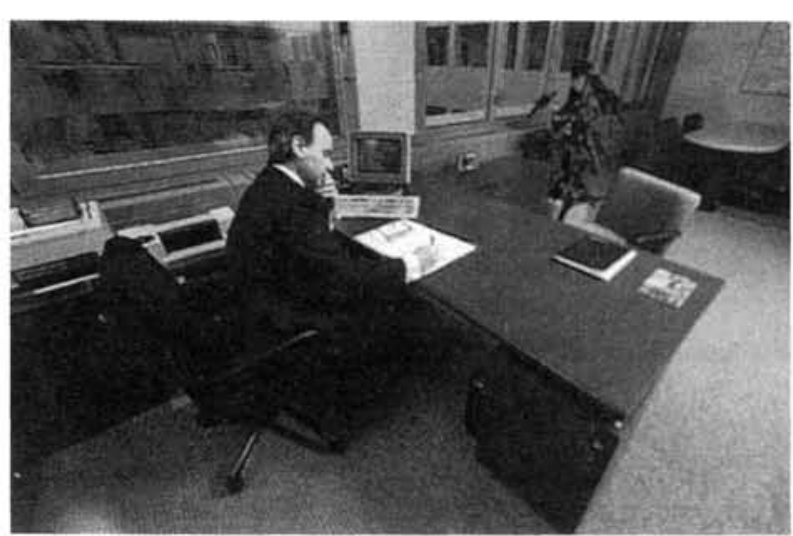

http://informesdelaconstruccion.revistas.csic.es 







\section{INTRODUCCIÓN}

Las instalaciones y centros de trabajo de una empresa constituyen uno de sus principales signos de identidad. La nueva oficina central de IBM España quiere servir, además, de muestra de lo que ofrece como entidad comercial y de lo que significa como creadora de una peculiar cultura de empresa. Innovación tecnológica y servicio al individuo son ideas centrales en la concepción de este edificio.

IBM mantiene el reto de estar en vanguardia de la innovación, objetivo que esta construcción pretende cumplir. El edificio incorpora un completo conjunto de sistemas que le dotan de las herramientas informáticas necesarias para automatizar procesos como el control de la climatización o la ingeniería de oficinas. Las comunicaciones, elemento fundamental para una empresa, han merecido una atención especial que se traduce en una evidente mejora de los intercambios de información.

El edificio, en su conjunto, desde su singular diseño arquitectónico hasta la amplia gama de servicios que ofrece, está pensado con precisión para ayudar al individuo, tanto a las personas que trabajan en él como aquellas que mantienen relación con IBM España. La planificación total del entorno ha permitido que las más modernas herramientas tecnológicas se conjuguen de manera armónica con las mejores condiciones laborales y ambientales. Su estructura modular sirve para lograr una gran flexibilidad interna, pero también para crear áreas más individualizadas e independientes.

Las características de este edificio responden fielmente a la preminencia otorgada a la dimensión humana en IBM, tanto dentro de la empresa como en sus relaciones hacia el exterior. Tecnología, información e, incluso, arte se unen para conseguir que prácticamente cada rincón cumpla una función precisa en el transcurrir diario de las personas que lo ocupan o visitan.

Con la silueta de gran barco recortada en el paisaje madrileño de esta nueva oficina central, IBM España ha querido crear un espacio absoluto en el que la tecnología y el diseño se pongan a disposición permanente del individuo.
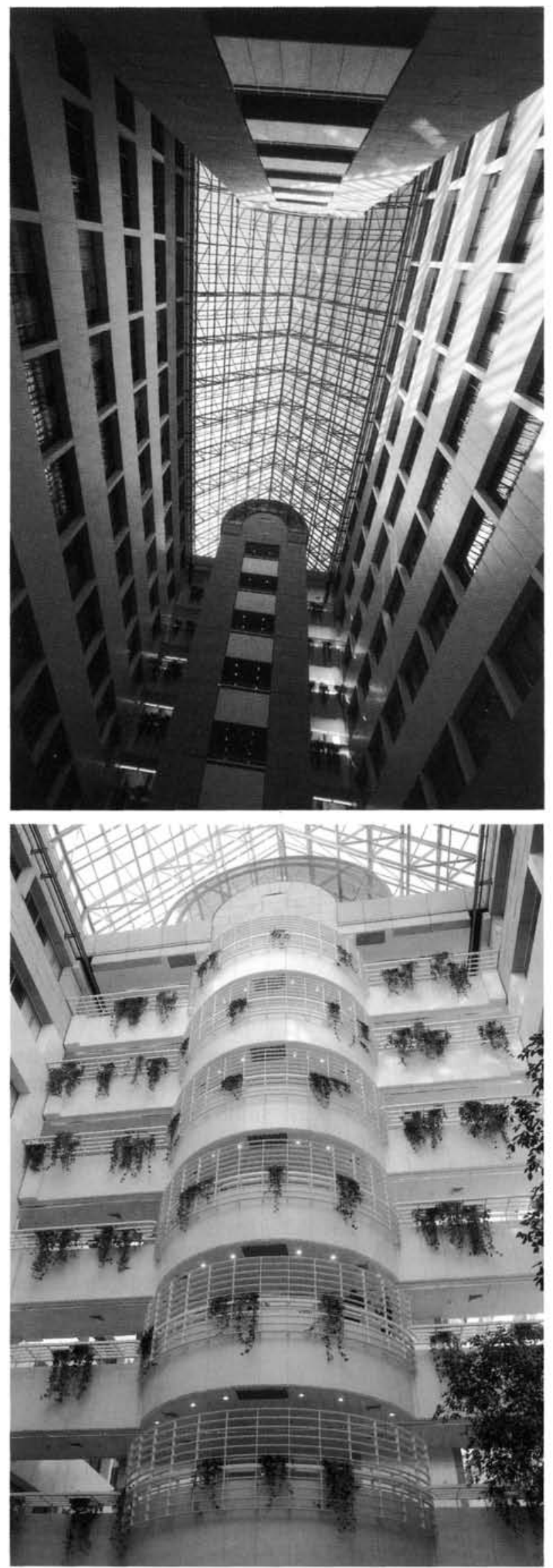

http://informesdelaconstruccion.revistas.csic.es 




\section{CARACTERISTICAS ARQUITECTÓNICAS}

Este edificio inteligente centraliza las operaciones comerciales de la compañía. Es capaz de prevenir numerosos problemas, de transmitir información, y de controlar sus necesidades y las de sus ocupantes.

Porque el edificio IBM está ideado para hacer más segura, confortable, rápida y eficaz la labor de los profesionales que en él trabajan. Así es la sede de las tecnologias integradas.

\section{Capacidad}

Su superficie es de algo más de $75.000 \mathrm{~m}^{2}$ construidos. Con una estructura de $150 \mathrm{~m}$ de longitud por $50 \mathrm{~m}$ de anchura. Tiene diez plantas y capacidad para albergar a 1.500 personas. Bajo el nivel del suelo se encuentran tres garajes que dan cabida a más de 900 automóviles.

\section{Arquitectura singular}

El proyecto del edificio cuenta con $50.000 \mathrm{~m}^{2}$ dedicados a oficinas y zonas de servicios comunes. El acceso se realiza a través de ascensores que dividen el edificio en 4 zonas, alrededor de tres patios cubiertos que permiten el paso de la luz natural.

\section{Arquitectura y materiales}

- Estructura: Hormigón armado, hasta la planta 5, con forjados reticulares. Pilares metálicos, desde el nivel 5 al 10.

- Fachadas: Zócalos de granito y paramentos de ladrillo, materiales tradicionales de la arquitectura madrileña. Ventanas de aluminio lacado, con acristalamiento doble de color.

- Despachos: Tabiquería de pladur, con aislamiento en fibra de vidrio y modulación de $90 \mathrm{~cm}$. 


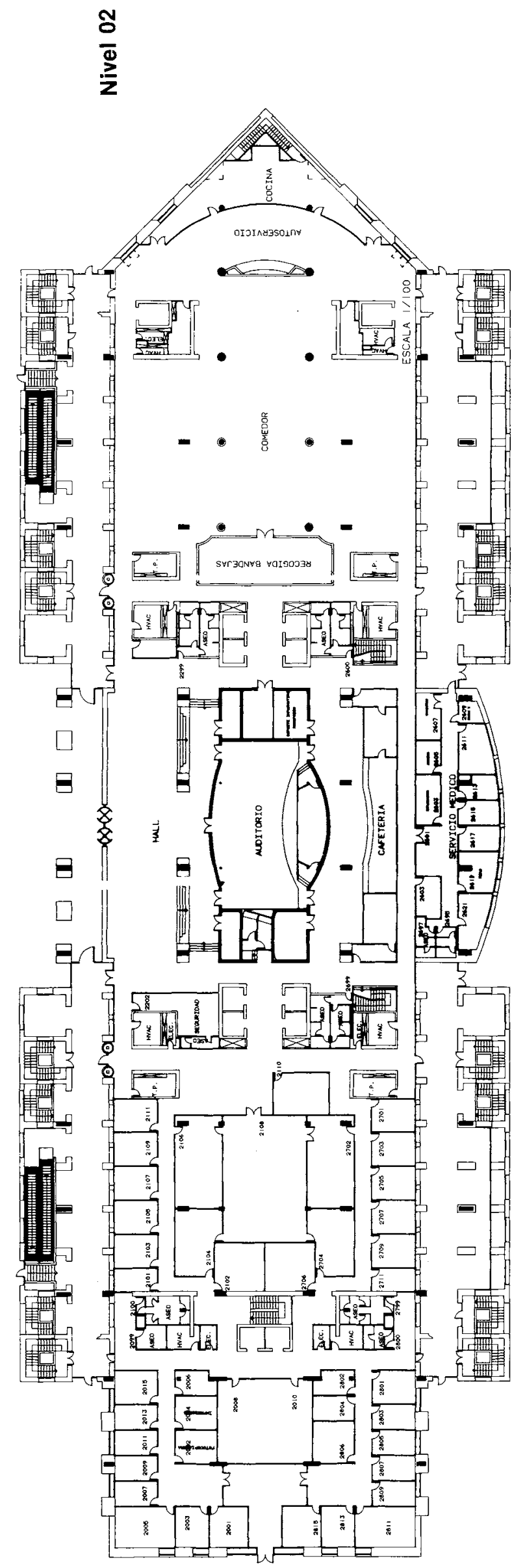

(c) Consejo Superior de Investigaciones Científicas Licencia Creative Commons 3.0 España (by-nc)

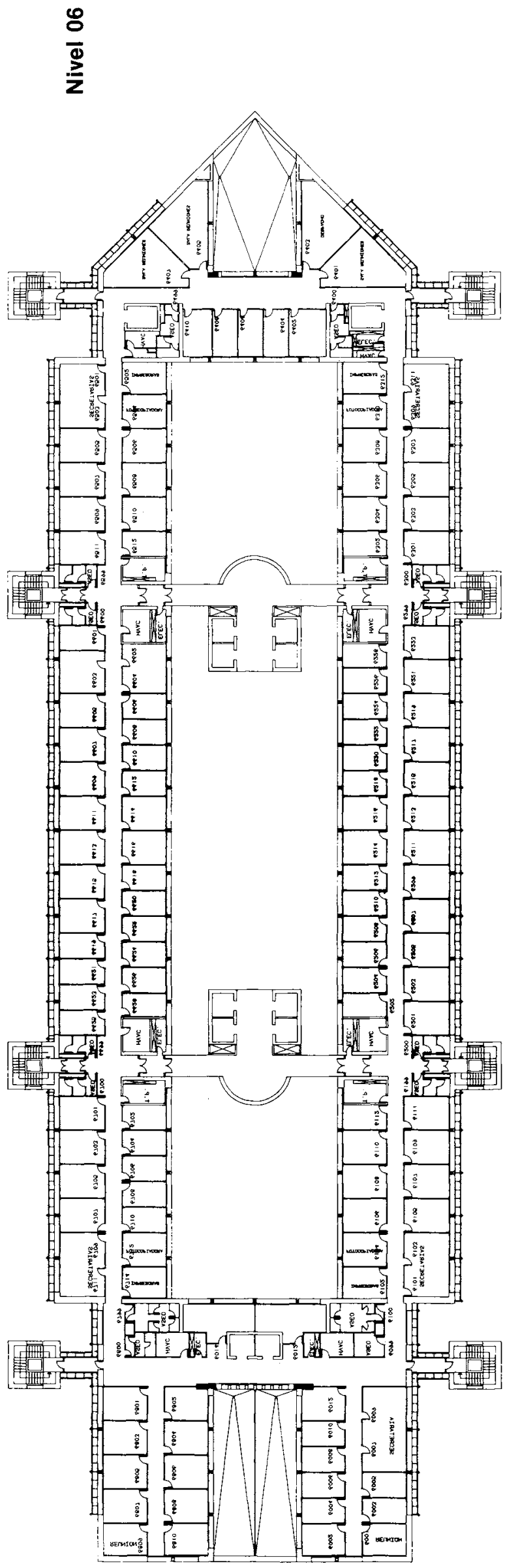

http://informesdelaconstruccion.revistas.csic.es 


\section{Algunas cifras}

-1.500 toneladas de granito.

- 5.500 toneladas de acero.

- 65.000 metros cúbicos de hormigón.

- 1.000 .000 de ladrillos.

- 10.000 metros cuadrados de vidrio.

- 40.000 metros cuadrados de tabiquería.

- 30.000 metros cuadrados de moqueta.

- 3.000 metros cuadrados de lucernario.

- 256 kilómetros de redes locales (LAN).

- $8.000 \mathrm{KVA}$.

-7 millones de frigorias.

\section{Concepción modular y flexible}

El edificio se divide en módulos de $90 \mathrm{~cm}$ cada uno, agrupados en despachos estándar de 3 y 4 módulos, que proporcionan espacios de 12 a 16 metros cuadrados.

Esta estructura modular permite una gran flexibilidad interna y sirve para crear áreas más individualizadas $\mathrm{e}$ independientes.

\section{Plena integración en su entorno}

La fachada exterior del edificio es de ladrillo y granito, acorde con el entorno de viviendas de los alrededores. Tampoco difiere de ellas por su altura.

\section{TRABAJAR EN EL EDIFICIO DEL MAÑANA}

La estructura del edificio IBM será, muy probablemente, común en un futuro próximo. Recibe el calificativo de "inteligente" porque lleva incorporada una red de comunicaciones capaz de transmitir toda la información utilizada en el edificio.
Y también porque cuenta con un sistema de seguridad y control de accesos adecuado a sus caracteristicas y uso.

Por otra parte, integra un control y regulación de las instalaciones de climatización, electricidad y protección contra incendio.

También incorpora servicios diversos de gestión como mantenimiento preventivo de instalaciones, inventario de material, planimetría, etc.

Por todo ello, este edificio es, sin duda, el edificio del mañana.

\section{Infraestructura de transporte y red de comunicaciones}

Este edificio se encuentra enteramente pre-cableado.

Esto significa que cada despacho dispone de una o dos placas multifuncionales, que proporcionan:

- Energía eléctrica.

- Conexión múltiple a las redes locales.

- Pares telefónicos.

Todo ello establecido cada dos módulos de $90 \mathrm{~cm}$. De esta forma cualquier usuario tiene siempre a su disposición varios de estos elementos. Para los $1.500 \mathrm{em}$ pleados, se han instalado más de 3.000 placas.

El sistema de cables ha sido diseñado para satisfacer varios objetivos:

- Soportar las tecnologías actuales y asegurar la conexión de terminales y ordenadores personales a los ordenadores centrales.

- Soportar las tecnologías futuras, con redes locales "token ring".

- Facilitar la redistribución de puestos de trabajo.

- Posibilitar, a lo largo del tiempo, la evolución de la tecnologia hacia sistemas futuros.

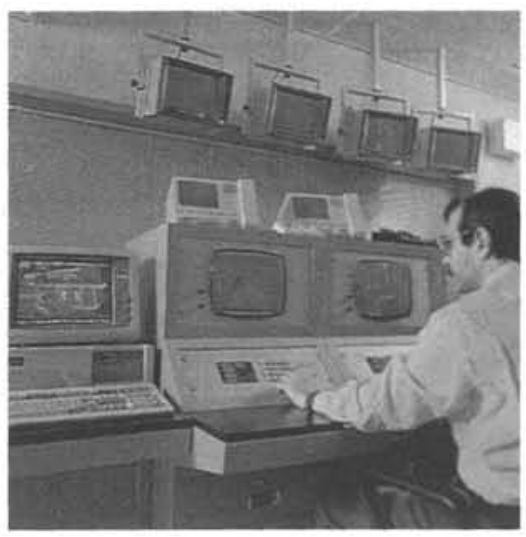

(c) Consejo Superior de Investigaciones Científicas Licencia Creative Commons 3.0 España (by-nc)
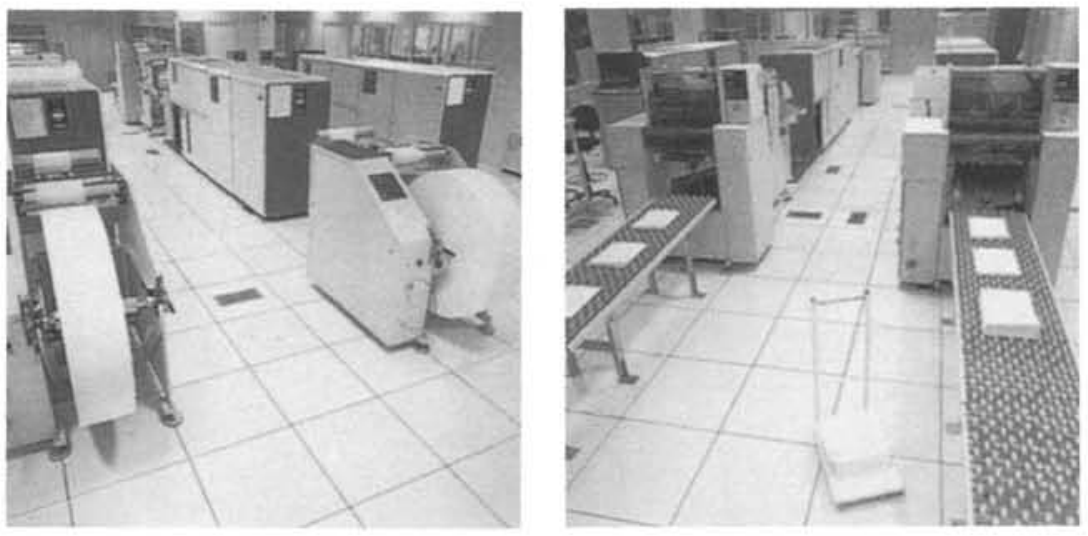

http://informesdelaconstruccion.revistas.csic.es 


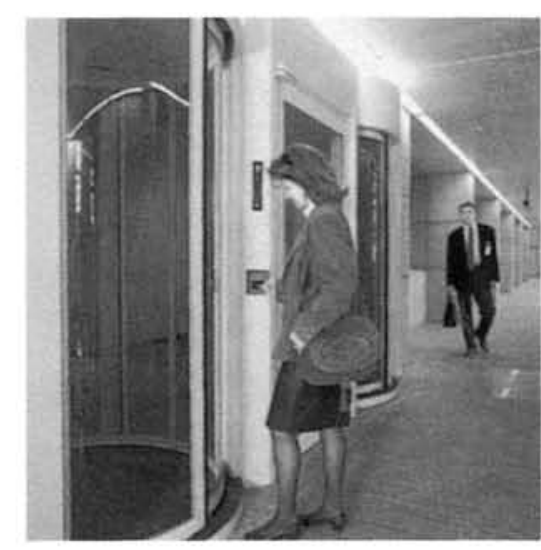

Este sistema se ha realizado en forma de anillos alrededor de cada planta. Otros anillos verticales aseguran un sistema de respaldo. La unión de ambos anillos, horizontales y verticales, se ha realizado a través de 4 centros de teleproceso por planta, que coinciden con los centros de gravedad del edificio.

Esta instalación incluye más de $256 \mathrm{~km}$ de cable de red que sirven a unas 5.450 tomas distribuidas por el edificio. Para gestionar el cableado, se ha desarrollado un "software" específico, que permite definir automáticamente la conexión necesaria para instalar un puesto de trabajo informático, y al mismo tiempo facilita las labores desconexión y reconexión cuando, por ejemplo, es necesario el traslado de un usuario.

\section{Control de las instalaciones de climatización}

Se ha instalado un sistema "inteligente" de calefacción, ventilación y aire acondicionado en el que un ordenador controla caudales y temperaturas.

Un sistema de sensores que transmiten la temperatura real de cada zona al ordenador de climatización permite establecer una ambientación personalizada en cada despacho.

\section{Sistemas de seguridad}

La protección contra incendios está garantizada por un sistema de rociadores de agua que se encuentran en los techos y que se dispararian automáticamente al producirse un fuego y alcanzar la temperatura crítica. El control se logra a través del sistema sensible de detección de humos. Este sistema se encuentra conectado al ordenador central, con alarma de zona en el centro de control de seguridad.

Los accesos al edificio están controlados por exclusas de entrada y salida, accionadas por lectoras IBM de tarjeta magnética.

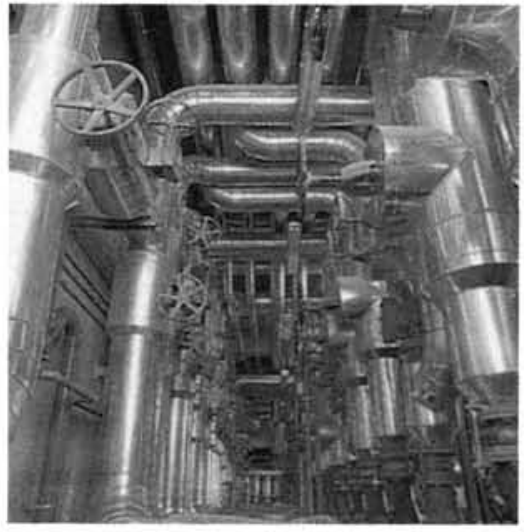

La misma tarjeta que utilizan los empleados para la identificación en el edificio.

Por otro lado cámaras de vídeo, situadas, tanto en el interior, como en los soportales y todas las fachadas del edificio, programables por zonas, controlan las partes del edificio.

\section{Audiovisuales y salas de reuniones}

Cuenta con más de 30 salas de conferencias, distribuidas por las distintas plantas, con una capacidad entre 10 y 150 personas.

Todas ellas están equipadas con proyector de transparencias y pantalla.

Por otro lado cuatro salas de exhibición y demostración de aplicaciones se sitúan en el nivel 1 y otras dos con una dedicación sectorial en el nivel 2. También, en la recepción principal se encuentra el auditorio, con una capacidad de 150 pesonas.

Dispone de toda clase de medios audiovisuales, incluida la doble proyección de transparencias, diapositivas y vídeo, con 2 PS/2 para realizar distintas presentaciones y demostraciones. Cuenta también con traducción simultánea.

\section{UN MICROCOSMOS AL SERVICIO DE SUS USUARIOS}

Tantas personas en un edificio único, requieren servicios comunes a todas ellas. Por eso la nueva sede de IBM cuenta con restaurante, con capacidad para 450 comensales y cafetería, banco, agencia de viajes, biblioteca..., centro de información y, naturalmente, un centro médico. Para garantizar un fácil acceso al Servicio Médico, éste se encuentra situado en la planta 2, al mismo nivel que la recepción. Para los casos de urgencia, tiene un acceso directo desde la calle, por medio de una rampa y puerta de emergencia. 